\title{
CASE OF SUBCLAVIAN ANEURISM
}

SUCCESSFULLY TREATED BY LIGATURE

\author{
$\mathbf{B Y}$ \\ H. H. CLUTTON, M.C. \\ SURGEON AND LECTURER ON SURGERY, ST. THOMAS'S HOSPITAL
}

Receired November 14th, 1896-Read May 25th, 1897.

THIs was a case of an aneurism of the third part of the right subclavian, for which the first part of the artery was ligatured twice, and on the second occasion was followed by a distal ligature to the first part of the axillary. A spare healthy man, aged 54, was sent to me by Dr. Sharkey in February, 1896, with the statement that so far as could be ascertained, by physical examination of his chest, the heart and aorta were free from disease.

He had an aneurism of the right subclavian in its third part, but no other evidence of arterial disease. His urine was normal, and there was no history of syphilis. His occupation was that of a cooper, which had necessitated an excessive use of the right arm and shoulder for the saw and hammer.

Three years ago after a long walk he had pain in the right shoulder, which he attributed to rheumatism. After a short rest the pain disappeared, but after any 
violent exercise it returned, and the intervals between the recurrences of pain became shorter. The pain, too, passed down the arm until it reached the tips of his fingers. He then tried rubbing his arm with oils, and used an electric battery; but finding the pain became worse he consulted a surgeon, who told him that he had an aneurism. This was in May, 1895.

On examination in February, 1896, a small sacculated aneurism was found in the course of the third part of the right subclavian artery, reaching from the outer border of the scalenus anticus to the clavicle. It was about the size of a large walnut. The pulsation of the right radial artery was occasionally smaller than the left, but not persistently so. He had no pain in the arm or hand so long as he was kept at rest.

As far as one could tell before the operation the aneurism did not extend beneath the scalenus anticus; and on the inner side of this muscle there was no difference between the two sides of the neck. It therefore seemed to be a very suitable case for the ligature of the second part of the subclavian, or at the junction of the first and second part.

On March 18th, 1896, an operation was undertaken for ligature of the subclavian, on the proximal side of the aneurism. The skin being drawn downwards, an incision was made from the sterno-clavicular articulation to the outer third of the clavicle. When the skin wound had been retracted upwards, the clavicular part of the sternomastoid muscle was completely divided. The internal jugular vein then came into view, and was retracted inwards. There was some little difficulty in defining the fibres of the scalenus anticus, as they appeared to be blended with the thickened fascia on the outer surface of the aneurism.

I felt it wiser, therefore, to do as Mr. Godlee had done in a similar case ('Med.-Chir. Trans,' vol. l $\times x v$, p. 275) namely, divide the scalenus anticus a little higher in the neck, above the aneurism, where the muscle could be 
distinctly defined from the external coat of the aneurism.

The phrenic nerve was easily recognised and drawn inwards with the internal jugular rein. When the scalenus anticus had been completely divided, it was seen that the aneurism really involved a portion of the second part of the subclavian, and that the ligature must be applied at the junction of the first and second part of this vessel. One branch was given off at this spot, and was directed upwards and backwards towards the neck. It was probably the deep cervical judging from its direction, and arose from the superior intercostal.

This artery was first ligatured; and then the subclavian on its proximal side, exactly at the inner margin of the scalenus anticus. Neither the transverse cervical nor the supra-scapular were seen; the former probably arose from that portion of the subclavian which was inrolved in the aneurism.

At the seat of ligature the artery appeared healthy.

The material used for the ligatures was carefully prepared goldbeater's skin, which had been kindly provided by Messrs. Ballance and Edmunds.

The aneurism needle was in each case first passed beneath the vessel and then threaded. A long ligature was drawn through the eye of the needle to its centre. The needle being withdrawn from beneath the vessel the loop was divided; two ligatures were thus left beneath the artery. These were then tied in the manner described by Messrs. Ballance and Edmunds as the "stay knot." Sufficient force was used to completely stop the pulsation in the parts beyond, but no attempt was made to divide the coats.

The wound was closed with horsehair sutures without. drainage, and without the employment of antiseptics; the surface was covered with sterilised gauze.

The first dressing was changed on the 23rd Marchfive days after operation,-as the temperature was not quite normal. Some sutures were removed, as there 
FIG. 1.

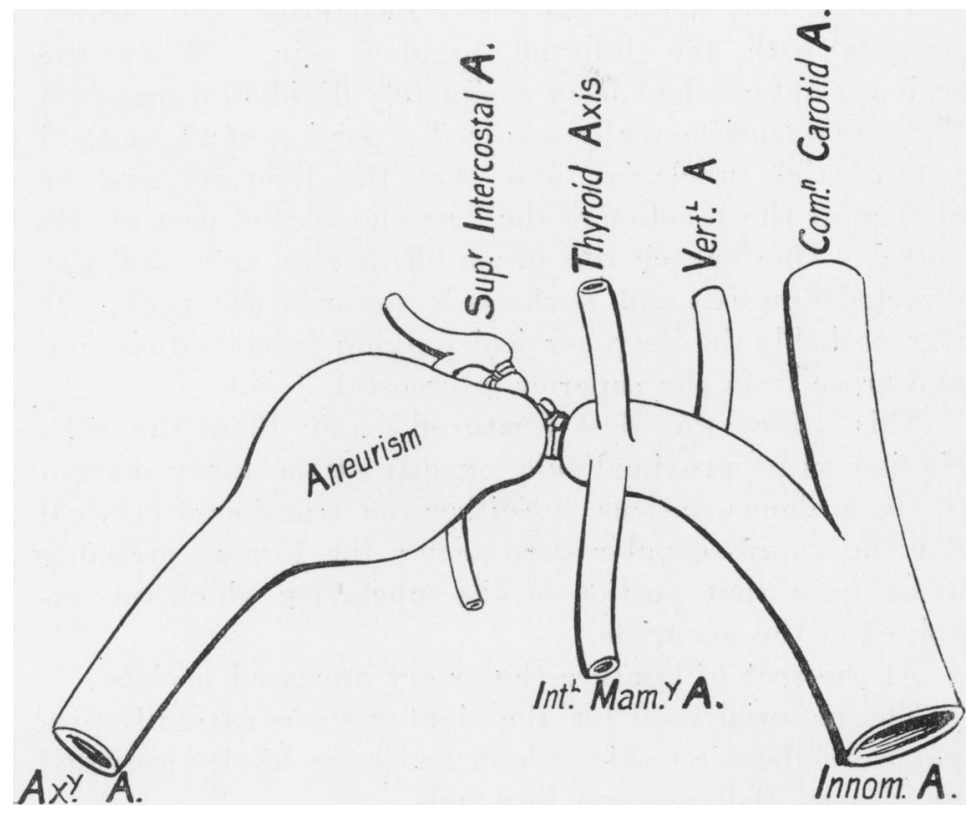

Diagram to show the seat of liguture in the tirst operation. Goldbeater's skin ligature.

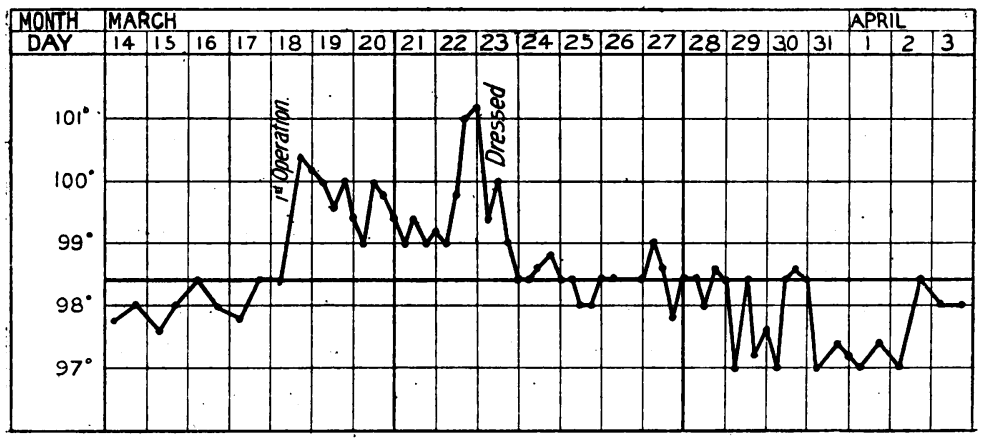

Temperature chart after first operation. 
was a little blood and pus beneath the incision; the suppuration, however, appeared to be quite superficial.

The wound was dressed again on the 25th, when the temperature was normal. Pulsation was now for the first time felt in the aneurism, but disappeared again a few days afterwards.

The suppuration at the surface of the wound continued for about ten days, but was quite insignificant in the amount of pus produced, and in its extent.

On April 7th there was still no pulsation in the aneurismal sac, but a bruit was detected.

On the 21st, after careful examination, pulsation in the aneurism was distinctly made out, but the radial artery could not be felt.

On the 28th, about six weeks after operation, both the aneurism and the radial artery could be felt pulsating as they did before the operation.

It was now cleur that our treatment had failed, either from absorption of the animal ligature or from its being tied with insufficient tightness, or possibly from a too brisk collateral circulation.

Digital compression of the highest part of the axillary artery produced no diminution in the force of the pulsation in the aneurism. This compression would probably exclude all the axillary anastomosis. I was forced, therefore, to conclude that either the ligature had been absorbed or that it had been insufficiently tied. Against the latter alternative was the fact that pulsation had been urrested at the time of the operation and was not felt till seven days afterwards, and that the pulsation again disappeared for two or three weeks, and was not distinct till five or six weeks atter operation. On the whole, therefore, I thought it most likely that the ligature had been absorbed.

Sepsis had, it is true, taken place at the surface of the wound, but so far as one could tell, the suppuration had not extended to any depth, and was soon over. If it had reached so far as the seat of ligature, it would 
account for premature softening of the ligature. But the artery was not pervious for five weeks, and another week elapsed before the pulsation was fully re-established. This is just about the time at which the animal ligature should disappear, and be replaced by young connective tissue. Messrs. Ballance and Edmunds have shown by their experimental work this to be about the time in an aseptic wound for the animal ligature to be replaced, and that if suppuration occurs the absorption of the ligature takes place still earlier. This raises the important question whether the animal ligature is trustworthy for large arteries where the blood pressure is considerable. For if sepsis was not accountable for the absorption of the ligature, it is clear that an animal material is not satisfactory for the ligature of a large artery in continuity-unless, indeed, there are still graver objections to the use of a silk ligature.

As the aneurism was now the same size as before the operation, I determined to re-ligature the subclaviun artery on the proximal side with silk. But I thought it best to wait a few weeks, if it were possible to do so. The man was therefore kept in the recumbent position, and a careful watch kept on the aneurism.

On June 8th, about ten days after the aneurism was recorded as pulsating as forcibly as before, it was noticed that it had perceptibly increased in size, and the man complained of pain down his arm. I decided, therefore, not to wait any longer.

On June 10th the second operation was undertaken. A curved incision was made above the scar of former operation, terminating just below the middle of the episternal notch. The clavicular part of the sterno-mastoid muscle was again divided, but at a higher level. The sternal origin of this muscle having been retracted inwards, the sterno-hyoid and thyroid muscles were exposed and divided.

The internal jugular vein and carotid artery were then seen lying one on each side of the centre of the wound, 
Frg. 2.

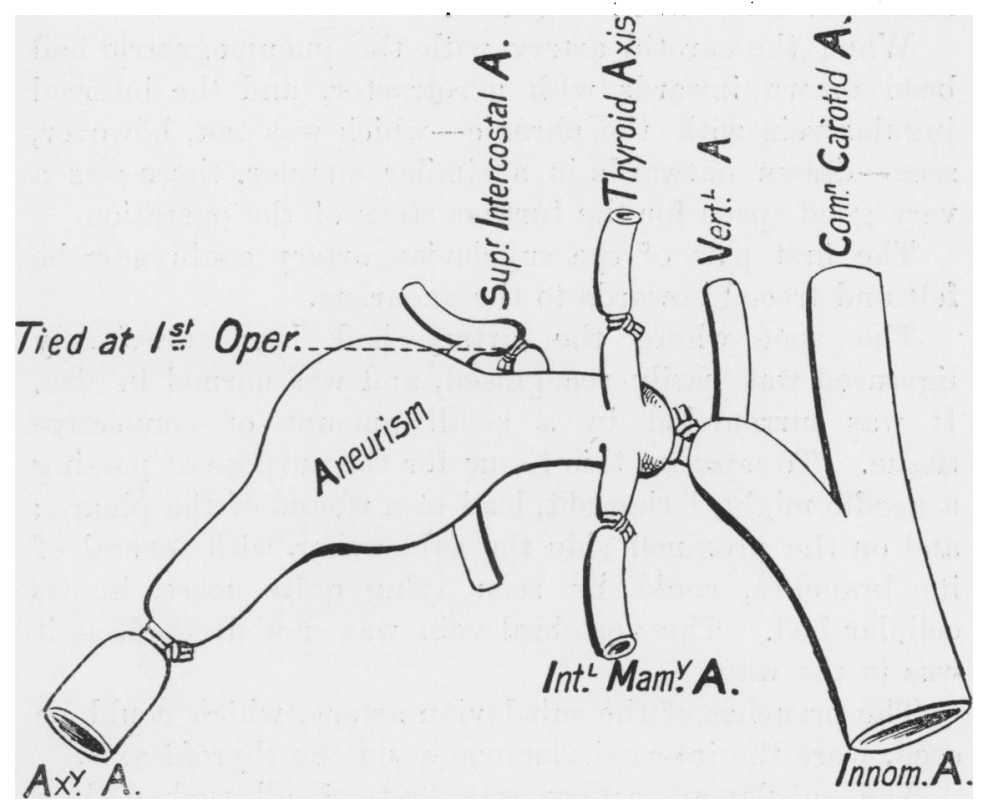

Diagram to show the ligatures applied in the second and third operations. Floss silk ligatures. The supra-scapular and transversalis colli are not shown, as they were not seen. It is impossible to say positively from which vessels they arose.

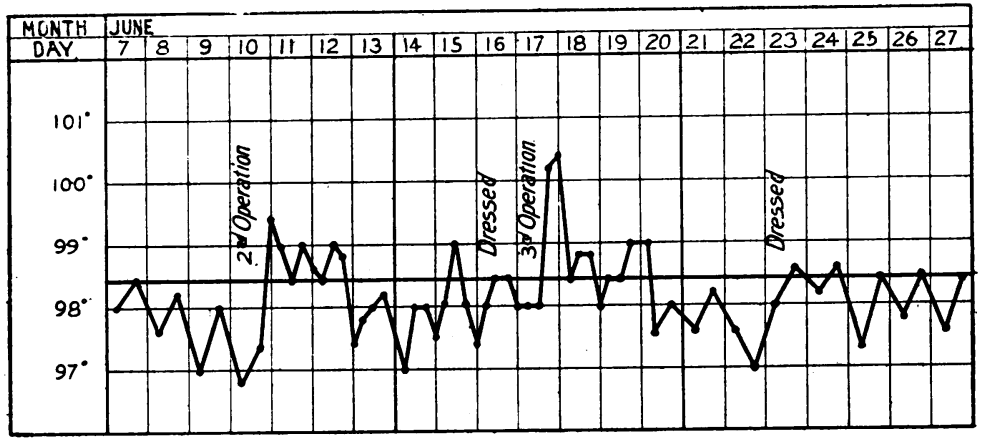

Temperature chart after second and third operations.

vOL. LXXX. 
which crossed them obliquely. The interval between these two structures was carefully enlarged, till the pneumogastric nerve was recognised.

When the carotid artery with the pneumogastric had been drawn inwards with a retractor, and the internal jugular vein with the phrenic-which was not, however, seen-drawn outwards in a similar manner, there was a very good space for the further steps of the operation.

The first part of the subclavian artery could now be felt and traced onwards to the aneurism.

The spot where the artery had been previously ligatured was easily recognised, and was normal in size. It was surrounded by a small amount of connective tissue. To separate this tissue for the purpose of passing a needle might, I thought, lead to a wound of the pleura; and on the proximal side the subclavian, with several of its branches, could be seen lying quite loose in its cellular bed. The vertebral vein was now divided, as it was in the way.

The branches of the subclavian artery, which could be seen, were the internal mammary and the thyroid axis.

The subclavian artery was first of all tied with a double floss silk ligature on the proximal side of these two branches, in the same manner as in the first operation. The thyroid axis and the internal mammary. were then similarly tied with the same material.

The pulsation in the aneurism, and in the radial artery, ceased as the ligature on the main vessel was being tightened, but no extra effort was made to rupture the coats.

The wound was closed without drainage, and without the use of any antiseptic solution, and dressed with cyanide gauze. The operation, which had occupied an hour, would have been a long and tedious business if there had been much inflammatory tissue as a result of the first operation. But, as has been shown, there was really very little-a fact which goes far to support the contention that the organisms present at the surface of 
the wound, after the first operation, had not penetrated to the deeper parts.

After the second operation the temperature remained normal, but the dressing was removed on the 16 th to see if the aneurism remained pulseless.

I had almost made up my mind at the second operation to tie the first part of the axillary as well as the subclavian, so as to cut off the collateral supply from the intercostal arteries, which might have been formed after the first operation. But on the whole I thought it better to wait a week to see the result of tying the subclavian with silk.

When, therefore, the dressing was removed I was not surprised to find the aneurism with distinct pulsation and bruit. The wound had healed, and the sutures were removed.

On the following day, June 17th, the third operation was undertaken, namely, the ligature of the first part of the axillary artery, so as to cut off the collateral supply from the distal side of the aneurism. An in. cision was made immediately below the clavicle, dividing the clavicular fibres of the pectoralis major. The artery was reached above the pectoralis minor, and tied as close under the clavicle as possible.

A double floss silk ligature was passed and divided. Then each was tied separately so as to avoid so large a knot as was made in the previous ligatures, but in tightening the first loops of the two knots the ends on each side were pulled simultaneously as in the "stay-knot."

The pulsation in the sac was materially diminished, but not entirely obliterated. Thinking there might be an enlarged abnormal branch between the aneurism and the scapula, such as the posterior scapular or suprascapular artery, I made an incision in this position. The supra-scapular nerve was seen, but no accompanying artery. A finger was also introduced which would have felt any enlarged vessel, but nothing of the kind was found. 
The wounds were closed without drainage or antiseptics, and dressed with cyanide gauze.

The temperature after this operation remained practically normal, but the dressing was changed on the 22nd to examine the aneurism. The wound was healed, and I thought there was no pulsation in the sac, but the next day, on re-examination, a very faint pulsation could be felt.

The subsequent history can be shortly told. The wounds healed by first intention; the aneurism began to diminish in size, and soon ceased to give even the faintest pulsation.

On July 13th I noted the fact that no pulsation had been felt for some days, and the aneurism was shrinking in size.

On August 3rd there was no swelling to be felt above the clavicle, so that the aneurism, by that date, may be said to have ceased to exist.

The patient went home on August 8th. Dr. Sharkey examined him before he left, and found no alteration in the physical signs in the chest. The pulse at the wrist had not returned, but there was no swelling in the arm or hand, and there was no pain or tenderness.

He was again seen in October, when the same condition existed, namely, complete absence of pulsation above the clavicle and no radial pulse at the wrist.

Remarks.-I do not wish to draw any definite conclusions from this interesting case, as it appears to me that there are not enough records as yet of operations upon subclavian aneurisms, which have been conducted on modern lines of treatment, to enable one to state, with any degree of accuracy, the particular method which is most likely to be successful.

I would like, therefore, to leave this case with as few observations as possible upon the details of treatment which I felt called upon to carry out during the progress of events which have been described.

For anyone who is interested in the history of the 
operative treatment of aneurisms of the third portion of the subclavian artery there is a very interesting article by Dr. Edmund Souchon, in the 'Annals of Surgery for 1895,' vol. ii, p. 545 and 743. Here also the treatment in the future is foreshadowed.

With regard to my own case I would summarise the details of the treatment in this way.

(1) There was sufficient room to apply a ligature on the proximal side of the aneurism, provided the coats of the artery were not divided by a tight ligature. If the latter method of applying a ligature were thought to be best, then the artery should be, in my opinion, divided between the two ligatures. The position of the artery to be tied in this case-namely, the root of the neckmakes this an undesirable method. The artery was not therefore tied with sufficient tightness to divide the coats in any of the operations recorded in this paper.

(2) An animal ligature appeared to me to be the best material, but the failure in this case of procuring occlusion of the vessel before the ligature disappeared, suggests that a more permanent material at the root of the neck would have been safer.

Some surgeons may say that if, with the animal ligature, the coats had been divided, such failure of procuring occlusion would not have occurred. But the large number of vessels arising in the immediate neighbourhood of the ligature, and the high blood pressure at this spot, induce me to think that an aneurism at the seat of ligature would not be an improbable accident as the result of the injured coats.

(3) It is probable that if the first ligature had been of silk instead of an animal material, the subsequent operations would have been unnecessary.

(For report of the discussion on this paper, see 'Proceedings of the Royal Medical and Chirurgical Society,' Third Series, vol. ix, p. 141.) 